\title{
OBSERVATIONS OF BRIGHTNESS OVER THE DISK OF THE QUIET SUN AT FREQUENGIES OF 85, 500 AND 1400 MG./S.
}

\author{
J. L. PAWSEY \\ Division of Radiophysics, Commonwealth Scientific and Industrial Research Organization, \\ Sydney, Australia
}

\begin{abstract}
Observations of radio emission from the quiet sun, which is believed to be due to thermal emission from the ionized gases of the solar atmosphere, provide information about the electron density and temperature throughout the atmosphere. Comprehensive information requires observations, preferably of the brightness distribution over the solar disk, over a large range of wave-lengths. Because such observations are both difficult and tedious it is desirable that a final model atmosphere should be based on a pool of observations taken by independent methods and observers. The following are recent contributions to this pool from the Radiophysics Laboratory.
\end{abstract}

\section{OBSERVATIONS AT 2 I GM.}

Christiansen and Warburton (1955) [1] have described observations of the brightness distribution over the solar disk at a wave-length of $21 \mathrm{~cm}$. (the continuum, not the hydrogen line). They used two multiple-element interferometers which scanned the sun strip-wise in various directions. They were able to recognize and reject contributions from the disturbed bright areas by means of the narrow peaks which these areas give on the records. The 'quiet sun' distribution was recognized as the lower envelope of a number of superposed records. The brightness distribution over the disk was deduced from an adaptation of the Fourier method described by O’Brien (1953) [2].

The derived quiet sun distribution is shown in contour form in Fig. I and corresponding radial brightness distributions in various directions in Fig. 2. The observations were taken between 1952 and 1954 and, as no substantial change was apparent during this interval, they probably refer to representative sunspot minimum conditions. The angular resolution of 284 
the observations varied between 3 and 4 minutes of arc and the results shown in Figs. I and 2 have been smoothed where necessary to correspond to a uniform 4 minutes. Limited angular resolution distorts the derived distribution to some extent and this factor is discussed in a paper by Smerd and Wild (paper 5I).

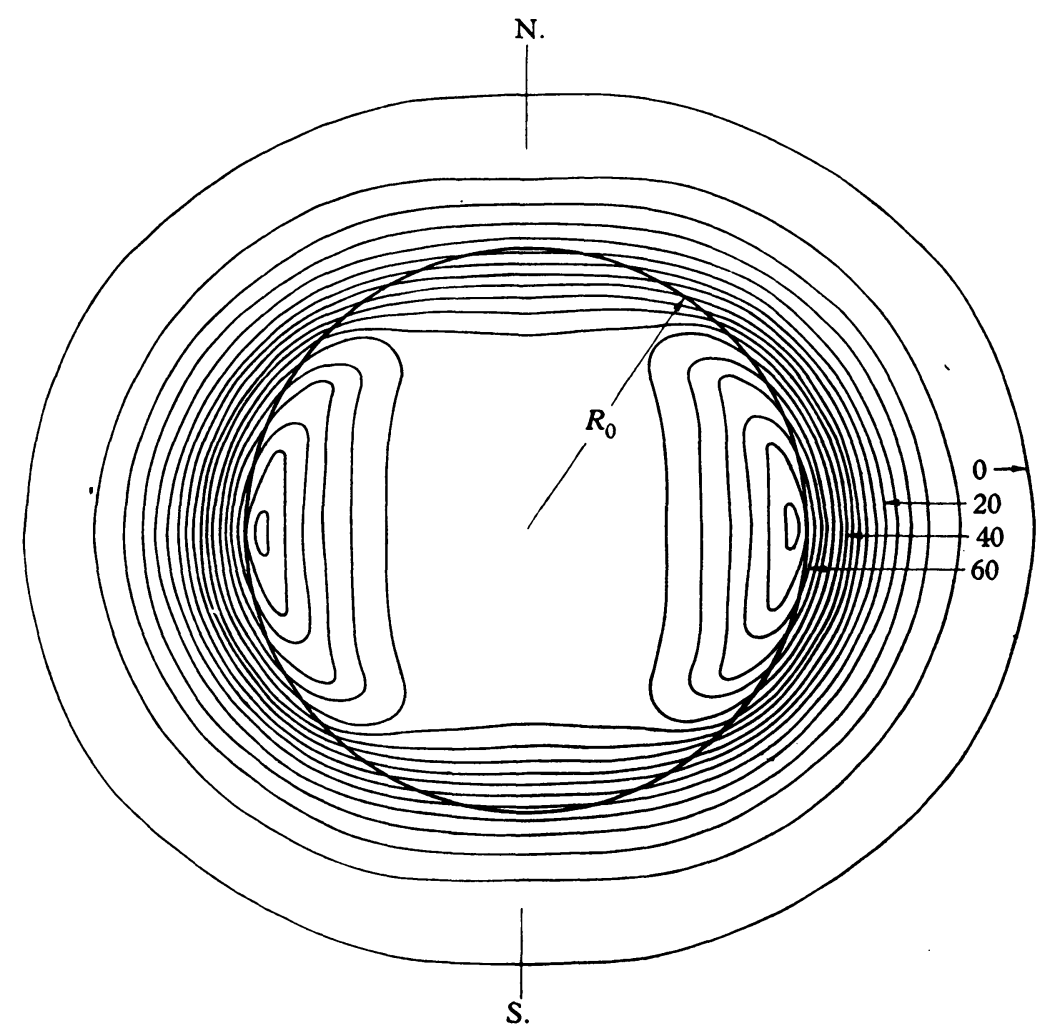

Fig. I. Derived $21-\mathrm{cm}$. radio brightness isophotes as observed with $4^{\prime}$ beam. Contour interval $4 \times 10^{3 \circ} \mathrm{K}$., central brightness temperature $4.7 \times 10^{4^{\circ}} \mathrm{K}$., maximum in peak $6.8 \times 10^{4}{ }^{\circ} \mathrm{K}$.

The most interesting feature of the distribution is the crescent-shaped bright areas at the east and west limbs. The northern and southern limits to these areas are also the places where the corona shows the typical changes in structure which differentiate the polar from the equatorial corona at sunspot minimum. 


\section{OBSERVATIONS AT 60 GM.}

Swarup and Parthasarathy (1955) [3] in Sydney adapted the original 32-element east-west Christiansen interferometer for use at a wave-length of $60 \mathrm{~cm}$. so that they could check Stanier's (1950) [4] results for the distribution over the quiet sun using the Christiansen-Warburton method. Stanier, using a multi-spacing two-aerial interferometer, had reached the surprising conclusion that the sun showed no limb brightening at this wave-length. At the same time in Cambridge O'Brien and TandbergHanssen (1955) [5] repeated the observations using Stanier's interfero-

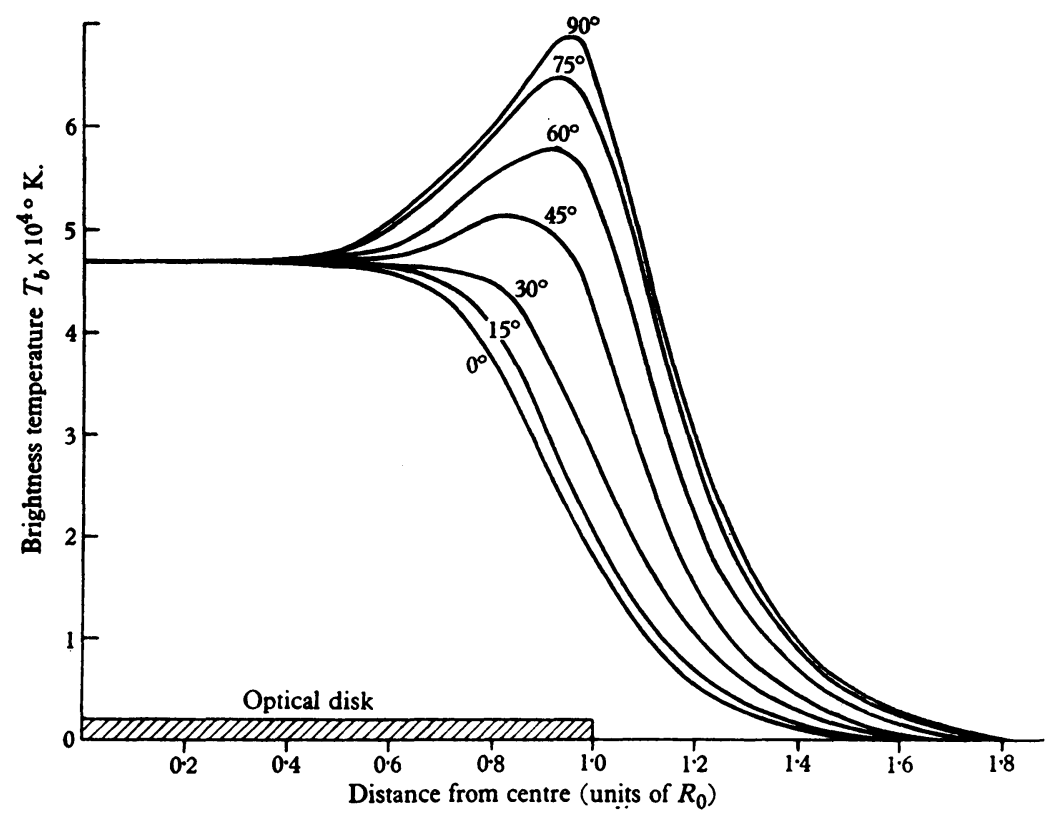

Fig. 2. Radial brightness distributions at $21 \mathrm{~cm}$. in various directions measured from pole of sun.

(Note that a corresponding figure published in Observatory in 1955 is slightly in error.)

meter method but extending it by using base lines in various directions. The Cambridge observations are the more complete because of the wide range of angles used and those authors have used them to derive a brightness distribution over the disk. However, the Sydney observations, being based on an independent method, give a valuable check.

Figs. $3 a$ and $b$ show a comparison between the Cambridge and Sydney observations for two directions of scan. The agreement is good and confirms the general distribution of brightness derived by the Cambridge workers. Both series conflict with the early Stanier results but it is not yet 


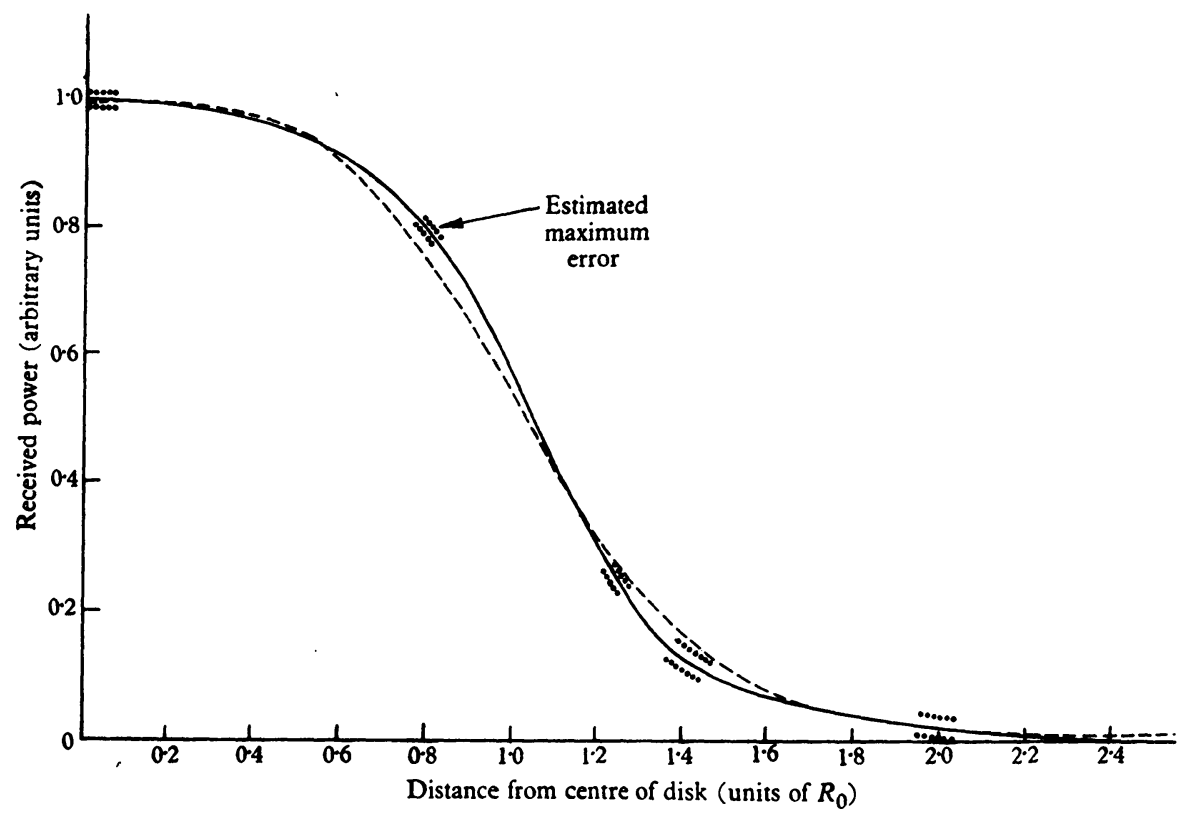

(a)

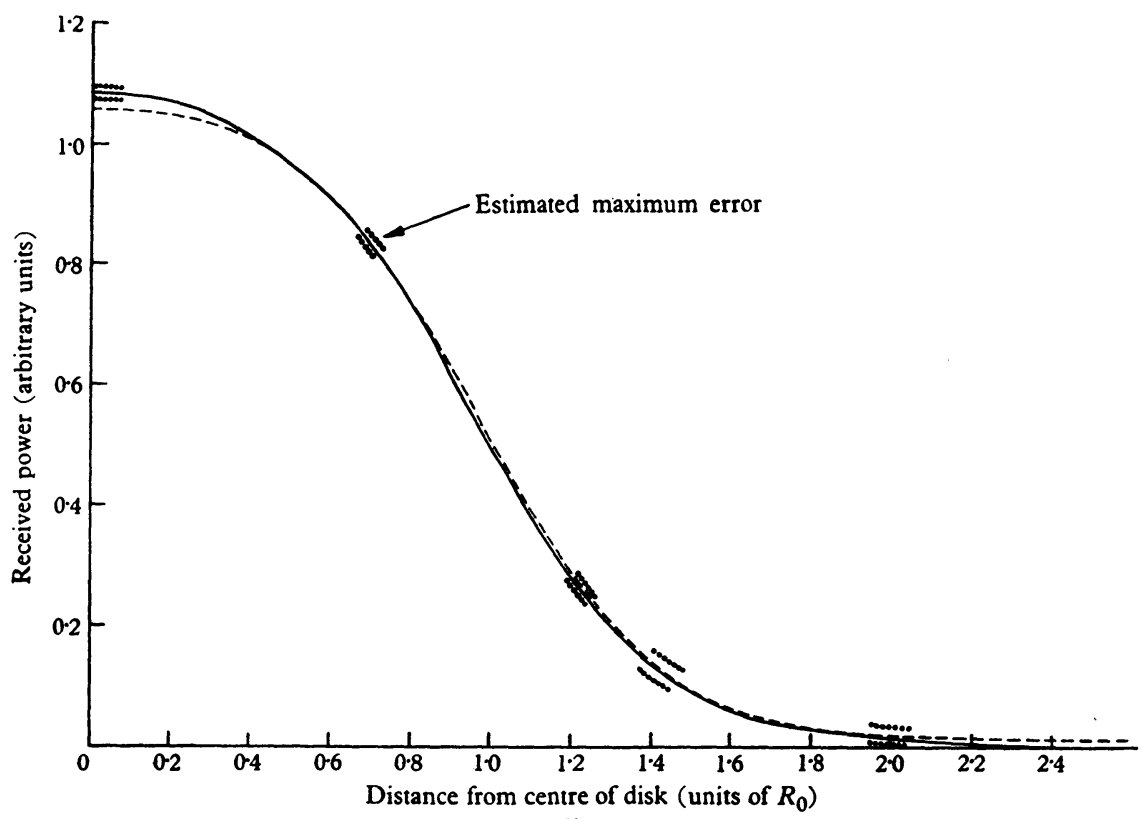

(b)

Fig. 3. Comparison of observed one-dimensional distributions (beam-width $8^{\prime}$ ) at $60 \mathrm{~cm}$. with those derived from O'Brien and Tandberg-Hanssen's two-dimensional distributions by smoothing with the aerial beam. (a) Scanning direction $90^{\circ}$ to solar axis; (b) $64^{\circ}$.

$$
287
$$


certain that the latter were in error since the difference might be due to changes associated with the changing phase of the sunspot cycle.

Turning to details, the Cambridge and Sydney observations show slight divergences which are believed to be outside the experimental errors of the latter. The form of the difference is that which would follow if the Cambridge observations were more 'smoothed' by unsuspected instrumental effects than the Sydney ones. The Sydney equipment had a resolution of 8 minutes of arc.

\section{OBSERVATIONS AT 3.5 METRES}

A series of observations of the sun were taken by A. G. Little in February and March I 955 using the 85 Mc./s. Mills Cross. This instrument has a pencil beam and in operation five traverses are made effectively simultaneously across the sun at declination intervals of $23^{\prime}$. Unfortunately the beam-width, $50^{\prime}$ in Right Ascension and from $5^{2}$ to $5^{\prime}$ in declination (the increase is due to foreshortening effects at low zenith angles) severely limits the derived information. The derived contours at times when the sun was quiet were approximately elliptical and sections through the centre exceeded the beam-width by ${ }_{1} 6^{\prime}$ in the east-west direction and $7^{\prime}$ in the north-south. It follows that the sun at 3.5 metres is substantially elongated in the east-west direction.

\section{REFERENCES}

[1] Christiansen, W. N. and Warburton, J. A. Aust. J. Phys. 8, 474, I955.

[2] O'Brien, P. A. M.N. R.A.S. 113, 597, 1953.

[3] Swarup, G. and Parthasarathy, R. Aust. J. Phys. 8, 487, 1955.

[4] Stanier, H. M. Nature, 165, 354, 1950.

[5] O'Brien, P. A. and Tandberg-Hanssen, E. Observatory, 75, I I, 1955.

\section{Discussion}

Ryle: Our measurements show that at $60 \mathrm{~cm}$. the Fourier components of the distribution are less than $\pm 1 \%$ from a spacing of $200 \lambda$ to $260 \lambda$. An analysis has been made both at this wave-length and at the other wave-lengths of $1.4,3.7$ and 7.9 metres to determine the possible errors caused by the finite resolving power used in the various measurements. The method used was to determine the difference between the adopted distributions, which in Bracewell and Roberts'* nomenclature are the 'Principal Solutions', and the unknown actual distributions. It can be shown that the difference or 'error distribution', if it exists, must have a periodic form having in each of the present measurements

* Bracewell, R. N. and Roberts, J. A. Aust. J. Phys. 7, 6r5, 1954. 
several maxima across the sun's disk. The difficulty of giving physical reality to the distributions found in this way gives considerable confidence in the distributions computed from the observations.

It is worth noting that the possible errors in the derived distribution depend on the amplitude of the highest order Fourier components obtained from the observations. The use of a moving aerial interferometer has in fact considerable advantages over a pencil-beam system of the same total aperture when used for examining a single source such as the sun, because all the Fourier components can be determined with equal weight; in the pencil-beam system the high-order components are necessarily severely attenuated.

Pawsey: The small differences which do exist between the Cambridge and Sydney observations indicate a tendency for the Sydney observations to show finer detail. For example, high-frequency Fourier components exist in the Sydney curves which the Cambridge observers measured but found negligible. Hence one or other of the series of observations must be slightly in error. The only conclusion I should like to draw at this moment is that it would be unwise to base theoretical conclusions on these details until better observations are available. 